\title{
All-Seeing-Access Sheath: A Novel Fluoroscopy-Free Placement Technique in Retrograde Intrarenal Surgery
}

\author{
Musa Ekici1, Berat Cem Ozgur3, Aykut Bugra Senturk1, Arzu Akdagli Ekici2, Cemil Aydin¹ and Mehmet Murat Baykam
}

\begin{abstract}
Objective: To evaluate the safety and effectiveness of fluoroscopy-free RIRS (Retrograde intrarenal surgery) method in urolithiasis.

Study Design: An observational study.

Place and Duration of Study: Department of Urology, Hitit University Corum Training and Research Hospital, Corum, Turkey, from October 2014 and October 2017.

Methodology: Preoperative and postoperative data of 62 cases of renal calculi that underwent fluoroscopy-free RIRS procedure by a single surgeon were prospectively evaluated. All manipulations were performed with a novel technique; under direct vision during the operation. Fluoroscopy device was kept at hand in the operating room, but was not used.

Results: The mean age was found as $51.73 \pm 12.63$ (22-82) years. Fortyone patients $(66.1 \%)$ were males and $21(33.9 \%)$ were females. The mean size of stones were $19.29 \pm 7.64(10-40) \mathrm{mm}$. The stone-free rate was calculated as $42(67.7 \%)$ cases in a single session one month after the surgery, and $15(24.2 \%)$ cases after the second session at the postoperative first month. A total of $57 / 61$ (91.9\%) patients were stone-free after the second procedure. No major intraoperative and postoperative complications were observed. As for the minor complications, one patient (1.6\%) developed hematuria and four patients $(6.5 \%)$ had fever.

Conclusion: The described fluoroscopy-free RIRS procedure can be performed effectively and safely in patients diagnosed with renal calculi, by endourologists.
\end{abstract}

Key Words: Renal calculi, Endourology, Fluoroscopy-free retrograde intrarenal surgery.

\section{INTRODUCTION}

Retrograde intrarenal surgery (RIRS) has become an effective and safe method in the treatment of renal calculi of various sizes over time. 1 This less invasive method is comparable with the other well known treatment techniques (percutaneous nephrolithotomy and open surgery), and high success were reported with low complication rates. ${ }^{2-5}$

As this tecnique has become widespread, several potential risks of radiation including cancer, and genetic mutations because of the use of fluoroscopy, should be discussed. Recent studies have aimed to use fluoroscopy at a minimal level to avoid damage to the healthcare staff and the patient in RIRS; but as the number of operations are rapidly increasing the cumulative doses of radiation is becoming a more

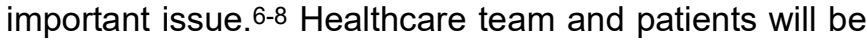
protected against detrimental impacts of fluoroscopy owing to retrograde intrarenal surgery technique

1 Department of Urology / Anesthesiology², Hitit University Corum Research and Training Hospital, Corum, Turkey

3 Department of Urology, Urology Clinic, Ankara Research and Training Hospital, Ankara, Turkey

Correspondence: Dr. Berat Cem Ozgur, Urology Clinic, Ankara Research and Education Hospital, Ankara, Turkey E-mail:bcemozgur@hotmail.com

Received: June 14, 2018; Accepted: October 29, 2018 performed without fluoroscopy under direct vision of the flexible renoscope via the guide, while complications seen with the methods performed blindly or with tactile sensation will be avoided.

In this study, the aim was to introduce and present success and reliability of all seeing access sheath method that decreases the intraoperative radiation and also avoids the blind insertion of ureteral access sheath (UAS).

\section{METHODOLOGY}

Cases of RIRS that were operated by a single surgeon at Department of Urology, Hitit University Corum Training and Research Hospital, Corum, Turkey, from October 2014 to October 2017, were prospectively evaluated. Patients with ureteral Double-J (DJ) inserted for dilatation, with anatomical anomalies, and who had previously undergone stone surgery, were excluded. A total of 62 patients, who were operated with this new method without using fluoroscopy, were included in the study.

All patients were informed about the procedure. Urinalysis and urine culture were performed. Patients who had a culture with growth were administered appropriate antibiotherapy, and the patients with sterile urine samples were then taken to the operation. Kidney Ureter-Bladder (KUB) study was ordered on the postoperative first day, and ultrasonography (USG) was 
taken of the patients with non-opaque stones. The patients who were not stone-free, were reevaluated with KUB and USG at the postoperative first month. The success status was considered as being stone-free both in KUB and USG, or presence of $<2 \mathrm{~mm}$ residual fragments. Complications and stone-free rates were noted.

All cases were operated under general anesthesia in the lithotomy position. First, a 9.5 Fr semi-rigid ureteroscope (Storz, Tuttlingen, Germany) was introduced, and lower end of the ureter was observed. The ureter was accessed through a guidewire (0.038-inch hydrophilic material coated flexible tip guidewire, CookMedical, Limerick, Ireland). The ureter was assessed for width and narrowing up to the last point where the semi-rigid ureteroscope could be advanced (preferably up to the renal pelvis).

If the ureter was wide enough, the flexible renoscope (Storz Flex-X2, Tuttlingen, Germany) was passed through the access sheath $(9.5 \mathrm{Fr}$, CookMedical, Bloomington, USA), and thus, the access sheath was attached to the flexible renoscope. Then, the flexible renoscope was introduced into the ureter via the guidewire under direct vision, and advanced to the kidney. The access sheath was advanced over the flexible renoscope with flexible renoscope functioning as a guide for the access, until the desired point. Thus, the patients and healthcare personnel were not exposed to radiation and protected against the harmful effects of fluoroscopy. Since the access sheath was inserted under direct vision rather than blindly or with tactile sensation, it was aimed to avoid luminal injuries.

Next, the stones were broken by laser (Sphinx 30 Litho, Holmium-YAG laser, pulse energy $0.5-4.0 \mathrm{~J}$, frequency single $4-20 \mathrm{~Hz}$, pulse peak power $15 \mathrm{~kW}$ ). At the end of the procedure, the flexible renoscope was pulled out from the ureter and kidney by observing the lower end in order to understand whether there was an injury in the ureter, and a $4.7 \mathrm{Fr} .26 \mathrm{~cm}$ DJ stent was routinely inserted.

Statistical analyses were carried out using SPSS Version 22.0, (SPSS Inc., Chicago, IL, USA; lisans, Hitit University) package software. Normality was studied with Kolmogorov-Smirnov tests. The descriptive statistics are expressed as mean \pm standard deviation for the continuous variables, and number and percentage for categorical variables. Parametric paired sample t-test was used for the comparison of preoperative and postoperative hematocrit and creatinine levels. Spearman's correlation coefficient was used to investigate the correlation between stone size and operational time. Chi-square test was used for comparison of stone-free rate according to the sessions. $\mathrm{P}<0.05$ value was considered statistically significant.

\section{RESULTS}

A total of 62 patients with a mean age of $51.73 \pm 12.63$ (22-82) years were included in the study with $41(66.1 \%)$ male and $21(33.9 \%)$ female subjects. The mean age was $49.83 \pm 13.50$ (22-82) years in male and 55.43 \pm 10.02 (30-70) years in female patients.

The mean stone size was found as $19.29 \pm 7.64(10-40 \mathrm{~mm})$ with $33(53.2 \%)$ stones localized in the right kidney and $29(46.8 \%)$ in the left kidney. The stone localizations were found as lower pole in $12(19.3 \%)$, pelvis in 42 $(67.7 \%)$, upper pole in five patients $(8 \%)$, and multiple calyces in three $(4.8 \%)$ patients.

The mean operational time was $52.34 \pm 18.33$ (20-85) minutes. The mean duration of hospitalization was 1.42 \pm 0.53 (1-3) days. Comorbidities were found as hypertension (HT) in $16(25.8 \%)$ and diabetes mellitus (DM) in five $(8 \%)$ patients. As complications; $4(6.5 \%)$ patients

Table I: The crosstabs of stone-free success according to the localization.

\begin{tabular}{|c|c|c|c|c|c|c|c|}
\hline & & \multicolumn{3}{|c|}{ Stone-free 1} & \multicolumn{3}{|c|}{ Stone-free 2} \\
\hline & & Incomplet ED & Complet ED & Total & Incomplet ED & Complet ED & Total \\
\hline \multirow[t]{2}{*}{ Right kidney pelvis } & $\mathrm{n}$ & 7 & 15 & 22 & 2 & 20 & 22 \\
\hline & $\%$ & 31.8 & 68.2 & 100.0 & 9.1 & 90.9 & 100.0 \\
\hline \multirow[t]{2}{*}{ Left kidney pelvis } & $\mathrm{n}$ & 5 & 15 & 20 & 1 & 19 & 20 \\
\hline & $\%$ & 25.0 & 75.0 & 100.0 & 5.0 & 95.0 & 100.0 \\
\hline \multirow[t]{2}{*}{ Right kidney lower pole } & $\mathrm{n}$ & 5 & 2 & 7 & 2 & 5 & 7 \\
\hline & $\%$ & 71.4 & 28.6 & 100.0 & 28.6 & 71.4 & 100.0 \\
\hline \multirow[t]{2}{*}{ Right kidney upper pole } & $\mathrm{n}$ & 1 & 4 & 5 & 0 & 5 & 5 \\
\hline & $\%$ & 20.0 & 80.0 & 100.0 & 0.0 & 100.0 & 100.0 \\
\hline \multirow[t]{2}{*}{ Left kidney lower pole } & $\mathrm{n}$ & 1 & 4 & 5 & 0 & 5 & 5 \\
\hline & $\%$ & 20.0 & 80.0 & 100.0 & 0.0 & 100.0 & 100.0 \\
\hline \multirow[t]{2}{*}{ Right kidney multiple calyces } & $\mathrm{n}$ & 0 & 1 & 1 & 0 & 1 & 1 \\
\hline & $\%$ & 0.0 & 100.0 & 100.0 & 0.0 & 100.0 & 100.0 \\
\hline \multirow[t]{2}{*}{ Left kidney multiple calyces } & $\mathrm{n}$ & 1 & 1 & 2 & 0 & 2 & 2 \\
\hline & $\%$ & 50.0 & 50.0 & 100.0 & 0.0 & 100.0 & 100.0 \\
\hline \multirow[t]{2}{*}{ Total } & $\mathrm{n}$ & 20 & 42 & 62 & 5 & 57 & 62 \\
\hline & $\%$ & 32.3 & 67.7 & 100.0 & 8.1 & 91.9 & 100.0 \\
\hline
\end{tabular}




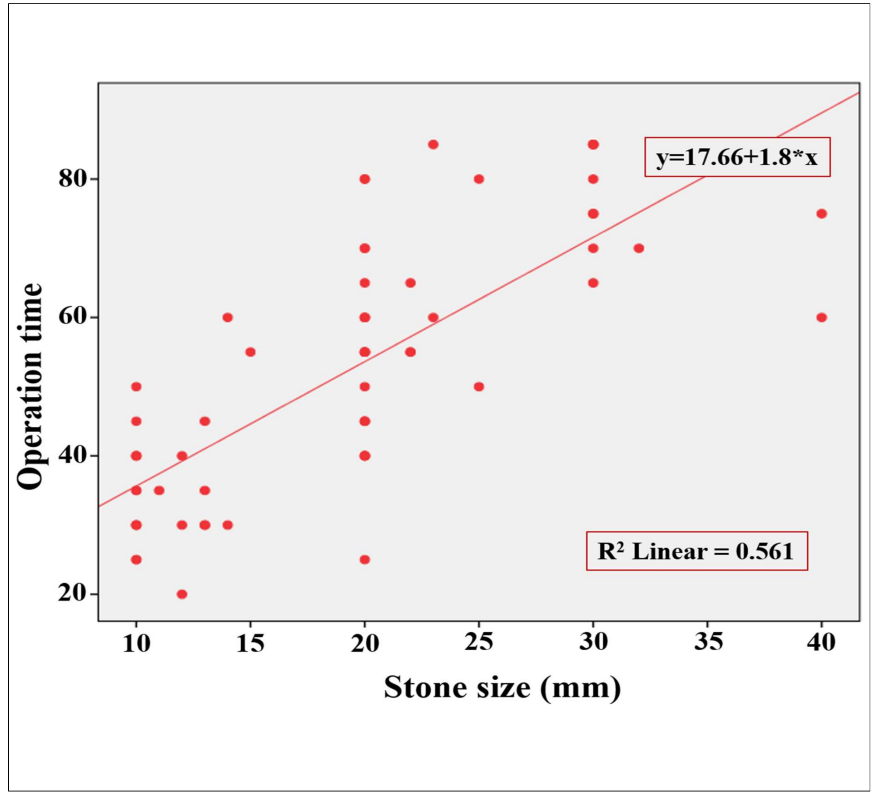

Figure 1: Scatter plot of the stone size and the operational time correlation.

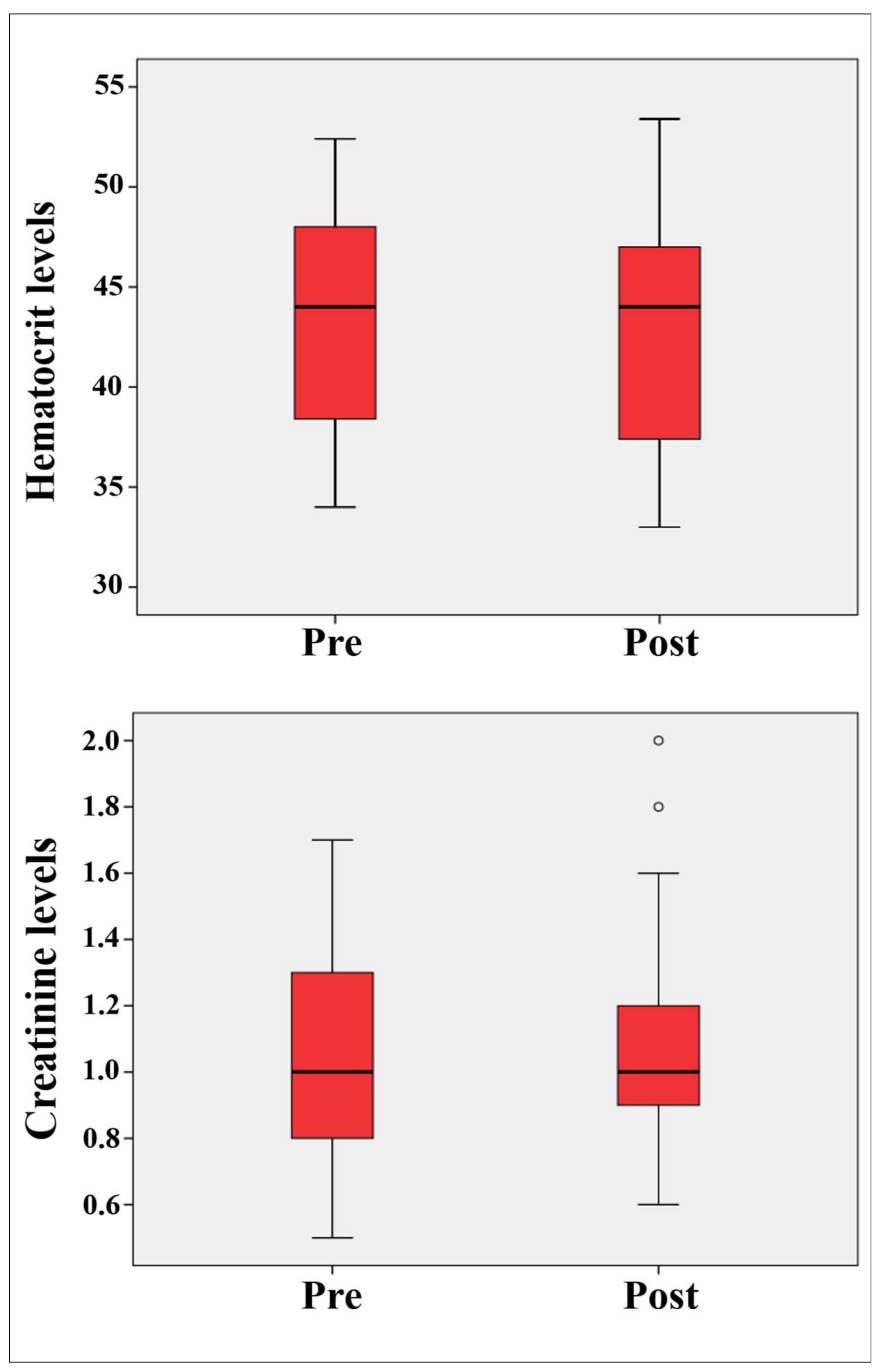

Figure 2: Box plot of the hematocrit and creatinine levels. developed subfebrile fever that lasts for 24 hours (Clavien grade 1) with an unknown etiology, and macroscopic hematuria was detected in one $(1.6 \%$, Clavien grade 2$)$. Hematoma and urethral injury were not observed in any patients.

DJ catheters were inserted in all patients after the procedure and intraoperative USG was performed in order to confirm that it was in the renal pelvis. All DJ catheters were observed to be intact on KUB ordered on the postoperative first day.

Stone-free rate was $67.7 \%(n=42)$ after the first session, and this rate was $91.9 \% \quad(n=57)$ after the second session. There was a statistically significant difference between these rates $(p=0.001)$. A more successful stone-free rate was achieved after the second session. The second operations were performed within fifteen days after detecting residual stone burden. Extracorporeal shock wave lithotripsy (ESWL) could also be offered as an option to some of these patients, but some patients' stones were non-opaque, and some patients preferred this treatment modality. Residual stones (presence of $<2 \mathrm{~mm}$ residual fragments) occurred only in five patients after the second session.

The success rate was lower in the lower pole stones. The success rates, according to the stone localizations, are presented in Table I. There was a high correlation between the stone size and operational time (Spearman's rho $=0.783 ; p<0.001)$. Scatter plot of this correlation is shown in Figure 1 with regression curve.

The mean preoperative hematocrit level was 43.24 \pm 5.41 and mean postoperative hematocrit level was $42.90 \pm 5.10$. No statistically significant difference was found between the pre- and postoperative hematocrit levels $(p=0.072)$. Moreover, no statistically significant difference was found between the pre- and postoperative creatinine levels $(1.053 \pm 0.27$ vs. $1.055 \pm 0,26)$ $(p=0.962)$. Box plot of the hematocrit and creatinine levels is given in Figure 2.

\section{DISCUSSION}

Fluoroscopy is an important tool, which should be available in the operating room in order to perform endoscopic interventions safely. ${ }^{8}$ In RIRS, this tool is used to provide access to the ureter, to define the renal collection system, to reach the stone, and to insert stents and guidewires. 9,10

In diagnosis and treatment of urinary stones, the use of fluoroscopy has become widespread, and thus patients and surgical team were exposed to higher amounts of radiation. ${ }^{11}$ The amount of exposed radiation depends on many factors such as the procedure performed, equipment at hand, and the technique used.12 The biological effects of this exposed radiation can be grouped as stochastic (random) and non-stochastic 
(deterministic) effects. Stochastic effect is related to the increase in doses rather than the severity of radiation; and at certain doses, certain predictable outcomes can be determined. Radiation-associated cancer and genetic impacts are stochastic. Surgeons use equipment such as lead apron, due to these effects, in order to minimize the radiation exposure. However, despite the use of these protective protocols, the surgeons, operation staff, and the patients expose to high doses of radiation during endourological procedures. ${ }^{13}$ Lesser use of fluoroscopy has become a current issue over time in order to protect both patients and the surgical team against such harmful effects of radiation.

A huge number of studies can be found in the literature on rigid and semi-rigid URS, which give insight to decrease the exposed radiation, as this procedure has been performed for decades. Fluoroscopy-free URS was performed by Mandhani et al. in patients with distal ureter stones. In that study with 110 patients, only 6 $(5.4 \%)$ patients required fluoroscopy. Reasons for the use of fluoroscopy were listed as fail to well localize the stone, presence of calcified ureteric stricture, and anatomical abnormalities. They concluded that to uphold the notion for radiation exposure to be as low as possible, fluoroscopy-free is a good option. ${ }^{14}$

There are limited number of articles on RIRS and fluoroscopy as compared to studies implicating rigid or semirigid ureteroscopes. In a study with 76 patients who underwent RIRS due to kidney calculi, reduced-radiation fluoroscopy was used. The guidewire was inserted visually by a rigid renoscope advanced up to the renal pelvis, and the access sheath was advanced with tactile sensation. The mean scopy time was found as $5.27 \pm 1.8$ seconds. Complications were reported in five (6.5\%) patients with urinary tract infection in two, and ureteral mucosal injury, hematuria and fever in one patient. ${ }^{15}$ In a study conducted on 140 patients who were diagnosed with kidney calculi and undergone RIRS, answer to the question "Could RIRS be performed without using fluoroscopy" was sought. The access sheath was advanced blindly with tactile sensation via the guidewire, and fluoroscopy was needed only in one patient. Five (3.5\%) patients developed minor complications and the stone-free rate was reported as $95.7 \%$. It was emphasized in the study that RIRS could also be performed fluoroscopy-free by experienced surgeons in uncomplicated cases. 16

In another study, Hsi et al. evaluated 162 patients who underwent RIRS. They utilized tactile sensation and endoscopic guidance in providing ureteral access, insertion of guidewire and DJ stent, and in other ureteroscopic parts of the procedure. They reported that fluoroscopy was not used in $117(75 \%)$ patients, and was used for less than two seconds only in $54 \%$ of the patients in order to confirm localization of the stent.
Fluoroscopy was used longer than 5 seconds in 17 $(11 \%)$ patients. The authors concluded that renoscopy can be used easily and safely without using fluoroscopy. ${ }^{3}$ But in all the studies mentioned above, access sheat is blindly inserted and that will probably cause ureteral problems.

Although UAS is known as a tool that minimizes the damage to the ureter in a study by Traxer, the ureteral wall injuries due to the use of UAS were assessed. ${ }^{7} \mathrm{~A}$ total of 359 cases were examined and it was found that UAS caused to ureteral wall lesions in 167 (46.5\%) patients. The cases were divided into 2 groups as low grade injuries (grade 0 - or 1 ) and high grade injuries (grade 2-3-4). Low grade injuries were observed in 311 $(86.6 \%)$ patients. Grade $-2-3$ and 4 injuries were reported as $10.1 \%, 3.3 \%$ and $0 \%$, respectively. It was stated in the mentioned study that insertion of DJ catheter before the RIRS surgery significantly decreased urethral injury. In the present study, patients who were inserted DJ catheters before RIRS for dilatation were excluded from the study, and yet no urethral injury was seen. This finding indicates that all seeing access sheath is a very safe method.

As is seen, in numerous studies blindly use of UAS without fluoroscopy caused many complications, while fluoroscopy was needed in some studies due to various reasons. Owing to this all-seeing-access sheath method, both the detrimental effects of fluoroscopy will be avoided and urethral injuries due to the use of UAS blindly and with tactile sensation will be prevented.

This study can be criticized for the advancement of the access sheath via the flexible renoscope may be damaging this brittle device, and may shorten its life. In a study, the factors prolonging device-life were listed as manipulations to be made correctly and carefully, keeping the device clean and correctly, the use of access sheath and avoiding excessive pressure on the deflexion mechanism. ${ }^{17,18}$

The average number of cases with one flexible renoscope is consistent with the literature, and according to these results, life of the device was not decreased with this technique. Normally, a compressive force is applied in order to pass the flexible renoscope through the access sheath, and the flexible renoscope is exposed to bending and distorsion due to its flexibility when inserted to the access sheath. In the presently described method, since the access sheath is glided over the flexible renoscope, it does not expose to this compressive force, bending and detorsion. When this method is applied in a larger number of cases, probably lifespan of the flexible renoscope will be increased.

Furthermore, treatment with this method does not affect the success rate. In general, stone-free rates after RIRS vary between $65 \%$ and $92 \%$ due to many factors like 
stone diameter, type, localization etc. ${ }^{19}$ The success was achieved in $42(67.7 \%)$ cases after the first session and in $57(91.9 \%)$ after the second session in the current study, which is consistent with the literature.

\section{CONCLUSION}

RIRS with all-seeing-access method has a short learning curve and can be performed safely and effectively by specialized surgeons under direct vision with the flexible renoscope functioning as the guide without using fluoroscopy, protecting the patients and healthcare personnel against harmful effects of radiation.

\section{REFERENCES}

1. Breda A, Territo A, Lopez-Martinez JM. Benefits and risks of ureteral access sheaths for retrograde renal access. Curr Opin Urol 2016; 26:70-5.

2. Mertoglu C, Bozkurt A, Keskin E, Gunay M. Evaluation of the effect of retrograde intrarenal surgery with myo-Inositol oxygenase. Pak J Med Sci 2018; 34:170-174.

3. Hsi RS, Harper JD. Fluoroless ureteroscopy: zero-dose fluoroscopy during ureteroscopic treatment of urinary-tract calculi. J Endourol 2013; 27:432-7.

4. Greene DJ, Tenggadjaja CF, Bowman RJ, Agarwal G, Ebrahimi KY, Baldwin DD. Comparison of a reduced radiation fluoroscopy protocol to conventional fluoroscopy during uncomplicated ureteroscopy. Urology 2011; 78:286-90.

5. Brisbane W, Smith D, Schlaifer A, Anderson K, Baldwin DD. Fluoroless ureteral stent placement following uncomplicated ureteroscopic stone removal: a feasibility study. Urology 2012; 80:766-70.

6. Mogilevkin $Y$, Sofer M, Margel D, Greenstein A, Lifshitz D. Predicting an effective ureteral access sheath insertion: A bicenter prospective study. J Endourol 2014; 28:1414-7.

7. Traxer $\mathrm{O}$, Thomas A. Prospective evaluation and classification of ureteral wall injuries resulting from the insertion of a ureteral access sheath during retrograde intra-renal surgery. $J$ Urol 2013; 189:580-4.

8. Geavlete P, Multescu R, Geavlete B. Health policy: Reducing radiation exposure time for ureteroscopic procedures. Nat Rev Urol 2011; 8:478-9.

9. Boulalas I, De Dominicis M, Defidio L. Semirigid ureteroscopy prior retrograde intrarenal surgery (RIRS) helps to select the right ureteral access sheath. Arch Ital Urol Androl 2018; 90:20-24.

10. Goldberg H, Golomb D, Shtabholtz Y, Tapiero S, Creiderman G, Shariv A, et al. The "old" $15 \mathrm{~mm}$ renal stone size limit for RIRS remains a clinically significant threshold size. World J Urol 2017; 35:1947-54.

11. Hein S, Schoenthaler M, Wilhelm K, Schlager D, Vach W, Wetterauer $\mathrm{U}$, et al. Ultra-low radiation exposure during flexible ureteroscopy in patients with nephrolithiasis - How far can we go? Urology 2017; 108:34-9.

12. Sfoungaristos S, Lorber A, Gofrit ON, Yutkin V, Landau EH, Pode $\mathrm{D}$, et al. Surgical experience gained during an endourology fellowship program may affect fluoroscopy time during ureterorenoscopy. Urolithiasis 2015; 43:369-74

13. Yecies T, Averch TD, Semins MJ. Identifying and managing the risks of medical ionizing radiation in endourology. Can J Urol 2018; 25:9154-60.

14. Mandhani A, Chaudhury H, Gupta N, Singh HK, Kapoor R, Kumar A. Is fluoroscopy essential for retrieval of low ureteric stones? Urol Int 2007; 78:70-2.

15. Kirac M, Tepeler A, Guneri C, Kalkan S, Kardas S, Armagan A, et al. Reduced radiation fluoroscopy protocol during retrograde intrarenal surgery for the treatment of kidney stones. Urol $\mathrm{J}$ 2014; 11:1589-94.

16. Peng Y, Xu B, Zhang W, Li L, Liu M, Gao X, et al. Retrograde intrarenal surgery for the treatment of renal stones: Is fluoroscopy-free technique achievable? Urolithiasis 2015; 43: 265-70.

17. Multescu R, Geavlete B, Georgescu D, Geavlete P. Improved durability of flex-Xc digital flexible ureteroscope: How long can you expect it to last? Urology 2014; 84:32-5.

18. Defido L, De dominicis M, Di gian Francesco L, Fuchs G, Patel A. Improving flexible ureterorenoscope durabilitiy up to 100 procedures. J Endourol 2012; 26:1329-34.

19. Oguz U, Balci M, Atis G, Bozkurt OF, Tuncel A, Halis F, et al. Retrograde intrarenal surgery in patients with isolated anomaly of kidney rotation. Urolithiasis 2014; 42:141-7. 